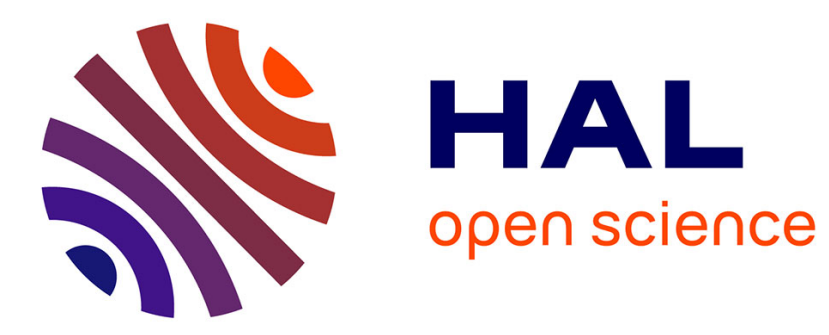

\title{
Under which conditions is character displacement a likely outcome of secondary contact?
}

\author{
R. Aguilée, B. de Becdelièvre, A. Lambert, D. Claessen
}

\section{To cite this version:}

R. Aguilée, B. de Becdelièvre, A. Lambert, D. Claessen. Under which conditions is character displacement a likely outcome of secondary contact?. Journal of Biological Dynamics, 2011, 5 (2), pp.135-146. 10.1080/17513758.2010.491559. hal-00607757

\section{HAL Id: hal-00607757 https://hal.science/hal-00607757}

Submitted on 10 Apr 2020

HAL is a multi-disciplinary open access archive for the deposit and dissemination of scientific research documents, whether they are published or not. The documents may come from teaching and research institutions in France or abroad, or from public or private research centers.
L'archive ouverte pluridisciplinaire HAL, est destinée au dépôt et à la diffusion de documents scientifiques de niveau recherche, publiés ou non, émanant des établissements d'enseignement et de recherche français ou étrangers, des laboratoires publics ou privés. 


\title{
Under which conditions is character displacement a likely outcome of secondary contact?
}

\author{
Robin Aguilée $^{a, *}$ and Benoît de Becdelièvre ${ }^{a, 1}$ and Amaury Lambert ${ }^{b, 2}$ \\ and David Claessen ${ }^{a, 1}$
}

Published in Journal of Biological Dynamics, 2011, 5(2): 135-146 with doi: $10.1080 / 17513758.2010 .491559$

\begin{abstract}
${ }^{a}$ Laboratoire Ecologie \& Evolution (UMR 7625), UPMC Univ Paris 06 - Ecole Normale Supérieure - CNRS, Paris, France.

${ }^{b}$ Laboratoire Probabilités \& Modèles Aléatoires, UPMC Univ Paris 06, Paris, France.

* Corresponding author. UMR 7625, Ecole Normale Supérieure, 46 rue d'Ulm, F-75230

Paris Cedex 05, France. Tel: +3314432 36 97. Fax: +331 443238 85. E-mail: robin.aguilee@biologie.ens.fr

${ }^{1}$ Ecole Normale Supérieure, CERES-ERTI, 24 rue Lhomond, F-75230 Paris Cedex 05, France. Tel: +331443227 21. Fax: +33144322727. E-mail: benoit.de.becdelievre@ens.fr; david.claessen@ens.fr

${ }^{2}$ UMR 7599, UPMC, 4 place Jussieu, Case courrier 188, F-75252 Paris Cedex 05, France. Tel: +331442785 69. Fax: +33 1442772 23. E-mail: amaury.lambert@upmc.fr
\end{abstract}

\section{Abstract}

Sympatric character displacement is one possible mechanism that prevents competitive exclusion. This mechanism is thought to be behind the radiation of Darwin's finches, where character displacement is assumed to have followed secondary contact of ecologically similar species. We use a model to evaluate under which ecological and environmental conditions this mechanism is likely. Using adaptive dynamics theory, we analyze different ecological models embedded in the secondary contact scenario. We highlight two necessary conditions for character displacement in sympatry: (i) very strong premating isolation between the two populations; and (ii) secondary contact to occur at an evolutionary branching point. Character displacement is then driven by adaptation to interspecific competition. We determine how ecological and environmental parameters influence the probability of ecological divergence. Finally, we discuss the likelihood of sympatric character displacement under disruptive selection in natural populations.

Keywords:

competitive exclusion; character displacement; ecological speciation; non-adaptive speciation; adaptive dynamics; Darwin's finches 


\section{Introduction}

The proposed scenario for the radiation of Darwin's finches on the Galápagos islands [14] hypothesizes the divergence of an ecological trait in two populations that came into secondary contact after a migration event $[31,17]$. This character displacement is thought to be a mechanism that prevents competitive exclusion. As a critical investigation of this scenario, we propose here a model to assess under which conditions the divergence of an ecological trait in two populations that are in sympatry is a likely evolutionary response to interspecific competition during secondary contact, thereby avoiding competitive exclusion.

The radiation of Darwin's finches is among the best documented examples of speciation [15]. The currently accepted speciation scenario hypothesizes that the speciation process is initiated in allopatry and completed in sympatry according to the three following steps. First, migrants colonize one island of the Galápagos archipelago from the mainland. Second, some individuals disperse onto another island and found a new colony. This step may be repeated several times. Third, migrants from a secondarily colonized island come into sympatry with the original colony. This secondary contact results in successful speciation if two conditions are fulfilled: (a) immigrant and ancestral populations do not interbreed and (b) they stably coexist, that is, no competitive exclusion [8]. Hereafter we refer to condition (b) as "stable coexistence" as it allows coexistence on an evolutionary time scale.

The second step of this speciation process (i.e., founding of new colonies) may ensure that condition (a) is satisfied. Indeed, finches use their songs - a culturally inherited trait - to discriminate between conspecific and heterospecific individuals. Consequently song variations should be selected against. Song can nevertheless change at dispersal events as a result of repetitive founder effects. In short, song divergence in allopatry induces premating isolation in sympatry $[10,11,12]$. Some morphological differences evolved in allopatry, on which mate choice is based, may also contribute to premating isolation in sympatry $[25,16,18]$.

On the contrary, condition (b) may be difficult to satisfy. One solution would be that migrants occupy an as yet unoccupied ecological niche. The previously cited studies $[14,15,17]$ however showed that secondary contact causes a divergence in beak size, allowing immigrant and ancestral populations to gradually feed on different resources. This implicitly suggests that no significant ecological divergence had occurred in allopatry. At the time of secondary contact immigrant and ancestral populations are still ecologically too similar to coexist. They share the same niche and thus one of the populations risks competitive exclusion. According to the accepted scenario, this is avoided because character displacement takes place, in sympatry, such that immigrant and ancestral populations form two different species that can stably coexist.

This speciation scenario - evolution of premating isolation in allopatry, followed by sympatry - has been termed "non-adaptive speciation" and has been proposed to be at work for other species too [29]. Rundell \& Price [28] suggested that this could be common in some groups of land snails, salamanders, lizards and plants. In each case, a pair of species is ecologically similar while in allopatry. Once in sympatry, competitive interactions result in competitive exclusion. In these cases the question is: can immigrant and ancestral populations avoid competitive exclusion by sympatric character displacement? A success would result in nonadaptive speciation.

We aim here to investigate the conditions under which this speciation scenario is possible. We will only model the key step of secondary contact. We assume premating isolation (possibly partial) of immigrant and ancestral populations. We ask under which 
ecological and environmental conditions stable coexistence (i.e. on an evolutionary time scale) can be established by character displacement in the two sympatric populations. In the context of adaptive dynamics theory [24, 9], and using numerical simulations, we analyze the secondary contact scenario for two different ecological models, assuming either two discrete resources or a continuum of resources. Our model differs from speciation models built using adaptive dynamics in that (possibly partial) reproductive isolation is already in place at secondary contact. This is a fixed parameter, assumed to have evolved in allopatry. We show that incomplete premating isolation strongly prevents character displacement. Moreover, character displacement is achieved only if populations are stuck at a fitness minimum (i.e., at an evolutionary branching point) at the time of secondary contact. Character displacement is then driven by disruptive selection. In these conditions, character displacement is analogous to evolutionary branching, without the need for positive assortative mating to evolve. We determine how other ecological and environmental conditions influence the probability of stable coexistence of the two populations. We finally discuss how the suitable conditions we found for character displacement are likely to be met in natural populations, and in particular in the Galápagos finches populations.

\section{Models}

\subsection{Secondary contact scenario}

We consider an initial resident (ancestral) population of $N_{0}$ individuals, monomorphic with ecological trait $u_{0}$. Due to a simple "quantitative genetics" rule for trait inheritance (see Section 2.2), the population is no longer monomorphic after a few generations. We let the resident population reach its ecological equilibrium, determined by the interaction with its dynamic food resources. We choose an ecological model such that the trait of the population converges under directional selection to a singular point $u^{*}$ [9] where the mutant invasion gradient vanishes (assuming $0<u^{*}<1$ ). Depending on our choice of parameter values, selection becomes either stabilizing or disruptive at this point. In the first case, the singular point is a fitness maximum called a "continuously stable strategy" (CSS): all mutants in a resident population at $u^{*}$ have a negative fitness, so that they cannot invade the resident population. Selection thus keeps the population at $u^{*}$. In the second case, $u^{*}$ is a fitness minimum called an "evolutionary branching point" (EBP): all mutants in a resident population at $u^{*}$ have a positive fitness, so that they could invade the resident population. At an EBP, an isolated large asexual population is expected to split into two ecologically diverging subpopulations, a phenomenon called "evolutionary branching". We assume that polygenic, diploid inheritance (hybrid formation) prevents evolutionary branching of the resident population, even when the resident is at an EBP. This is achieved by assuming a simple "quantitative genetics" rule for inheritance (see Section 2.2), which effectively prevents the population from evolutionary diversification.

Once the resident population is at its ecological equilibrium, $N_{m}$ migrants are introduced. Immigrants do not interbreed with residents. In case of partial premating isolation, this can nevertheless happen with low probability $1-\pi$; parameter $\pi$ is defined as the level of premating isolation. Immigrants have a trait drawn from a Gaussian distribution with mean $u_{r}+d_{u}$ and standard deviation $\sigma_{m}$, where $u_{r}$ is the mean trait of the resident population at the time of migration and $d_{u}$ is the ecological difference between the resident and immigrant populations. Migrant and resident populations are indeed assumed to derived from the same ancestral population, as this is the case for Darwin's finches speciation scenario. The migrant population can have experienced slightly different environmental conditions, resulting in an ecological difference $d_{u}$. 
A migration event can result in three outcomes: immigrants can either coexist with residents (i.e. absence of competitive exclusion), or go extinct, or replace the residents. If they go extinct, we let the resident population go back to its ecological equilibrium; we then reintroduce migrants. We repeat migration events until successful stable coexistence; or until an arbitrary maximum time limit of 2 millions generations is reached. When immigrants replace the residents, the immigrant population is considered as the new resident population and migrants are reintroduced in the same way as previously described.

\section{$2.2 \quad$ Ecological models}

The deterministic version of the first ecological model we use is exactly identical to that of Claessen et al. [4]. Let us sum up the main assumptions. Two resources of density $F_{1}(t)$ and $F_{2}(t)$ are available for a population of density $N(t)$. The ecological trait $u$ under evolution is the fraction of time spent foraging on resource $1(0 \leq u \leq 1)$. Let $A_{1}(u)$ and $A_{2}(u)$ be the search rate on the two resources, i.e. the volume cleared of resource per time unit per individual with trait $u$. The foraging ability on a resource is assumed to depend linearly on the time spent foraging on this resource, an assumption that mimicks the effect of learning or phenotypic plasticity [4]:

$$
\left\{\begin{array}{l}
A_{1}(u)=a_{1}+b_{1} u \\
A_{2}(u)=a_{2}+b_{2} u
\end{array}\right.
$$

The case $b_{1}>0$ and $b_{2}<0$ corresponds to situations such as learning ("strong trade-off" on resources, Figure 1). When $b_{1}=b_{2}=0$ there is no trade-off on resources, search rates are independent of $u$. The case $b_{1}<0$ and $b_{2}>0$ means that the foraging ability on a resource decreases with the time spent foraging on this resource; it is biologically less likely than the other cases. Search rates are necessarily positive for all $u$, thus $a_{1}$ and $a_{2}$ are positive and $a_{1}+b_{1}>0$ and $a_{2}+b_{2}>0$. Assuming that the per capita birth rate $\beta(u)$ is proportional to the consumption rate, we have:

$$
\beta(u)=k_{1} F_{1}(t) A_{1}(u) u+k_{2} F_{2}(t) A_{2}(u)(1-u)
$$

where $k_{1}$ and $k_{2}$ are the efficiencies of converting food into offspring.

The resources follow a semi-chemostat dynamics. Assuming a monomorphic population of trait $u$, the dynamics of the system is described by:

$$
\left\{\begin{array}{l}
\frac{\mathrm{d} N}{\mathrm{~d} t}=(\beta(u)-d) N(t) \\
\frac{\mathrm{d} F_{1}}{\mathrm{~d} t}=\delta_{1}\left(K_{1}-F_{1}(t)\right)-F_{1}(t) N(t) A_{1}(u) u \\
\frac{\mathrm{d} F_{2}}{\mathrm{~d} t}=\delta_{2}\left(K_{2}-F_{2}(t)\right)-F_{2}(t) N(t) A_{2}(u)(1-u)
\end{array}\right.
$$

where $d$ is the constant per capita death rate, and $\delta_{1}$ and $\delta_{2}$ are the degradation rates of the two resources $\left(\delta_{1} K_{1}\right.$ and $\delta_{2} K_{2}$ are the renewal rates).

For finite populations, we use a stochastic version of this model. Resources density is assumed to be in quasi-steady state with the current consumer population as in Claessen et al. [4]. The number of individuals depends on a scaling parameter $V$ ('volume'). We use a birth and death process in continuous time to simulate the evolution of the population. Opposite to Claessen et al. [4], we assume sexual reproduction. Mating pairs are randomly formed according to the premating isolation pattern, and reproduce according to individual birth rates. Offspring trait is drawn from a Gaussian distribution with mean the parental traits arithmetic mean and standard deviation $\sigma_{b}$. We simulate this way the quantitative genetics of the transmission of a polygenic trait. Neither environmental effects nor dominance effects on the ecological phenotype of the offspring are assumed. 

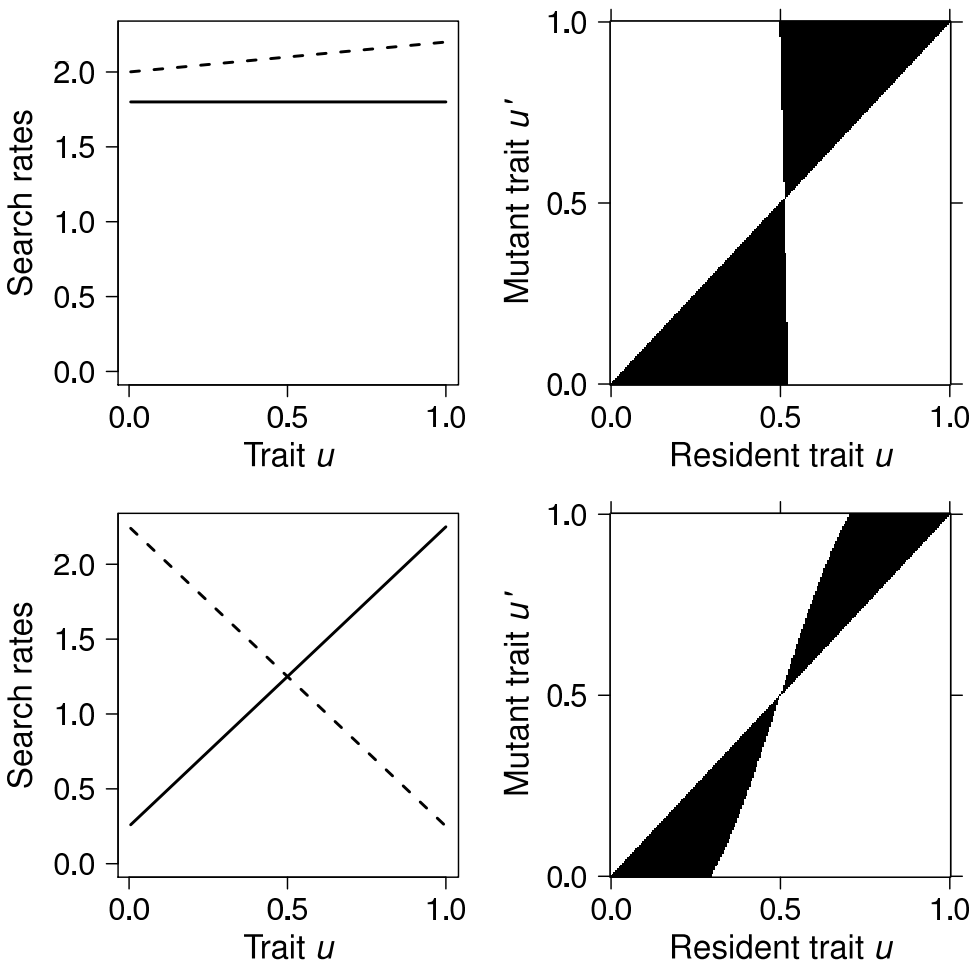

Figure 1: Trade-off on resources. Left panel: search rates (Equation 1) $A_{1}(u)$ on resource 1 (solid line) and $A_{2}(u)$ on resource 2 (dashed line) vs fraction $u$ of time spent foraging on resource 1 . The top panel shows a situation with a weak trade-off on resources $\left(a_{1}=1.8, b_{1}=0, a_{2}=2\right.$, $b_{2}=0.2$ ), the bottom panel illustrates a situation with a symmetric strong trade-off on resources which mimicks e.g. learning $\left(a_{1}=0.25, b_{1}=2, a_{2}=2.25, b_{2}=-2\right)$. Right panel: pairwiseinvasibility plots (PIPs) with the above trade-offs and $K_{1}=K_{2}=2, k_{1}=k_{2}=1, \delta_{1}=\delta_{2}=1$, $d=0.1$. White (resp. black) areas indicate positive (resp. negative) invasion fitness of a rare mutant with trait $u^{\prime}$ - i.e. its per capita growth rate - in a the resident population of trait $u$ at its ecological equilibrium (i.e. along a vertical line). Evolutionary trajectories of the ecological trait can be predicted using PIPs: the population is assumed to evolve by the successive replacements of the resident population by slightly different mutants with positive fitness. In the example with a weak trade-off (top panel), the point $u^{*}=0.5$ is a CSS. At this point, all mutants have a negative fitness (i.e. this is a fitness maximum, selection is stabilizing), hence they cannot invade the resident population which should remain on this trait value. In the strong trade-off example (bottom panel) the point $u^{*}=0.5$ is an EBP. At this point, all mutants have a positive fitness (i.e. this is a fitness minimum, selection is disruptive): an isolated population could split into two different ecological branches. The way we model sexual reproduction however prevents a single population from evolutionary branching.

Under stabilizing selection (CSS), the phenotypic variance of the offspring distribution is expected to decrease, whereas it is expected to increase under disruptive selection (EBP). However, near evolutionary singular points, selection is weak: the variance of the offspring distribution then changes very slowly compared to the population average. As a result, we consider $\sigma_{b}$ as constant. When premating isolation is not complete $(\pi<1)$, offspring type (resident or immigrant) is sexually inherited. At each birth event, mutation occurs with probability $\mu$, and modifies the offspring trait by a value drawn from a Gaussian distribution with mean 0 and standard deviation $\sigma_{\mu}$. Offspring sex-ratio is balanced.

Using the adaptive dynamics framework, Claessen et al. [4] showed that the deterministic version of the model has a convergent singular point $u^{*}$ which is an EBP if $b_{1} /\left(a_{1}+b_{1}\right)>b_{2} / a_{2}$ (strong trade-off on resources), and a CSS otherwise (weak tradeoff on resources) (Figure 1). In their asexual stochastic version of the model, they get 
evolutionary branching in an isolated population at an EBP. On the contrary, our sexual stochastic version does not allow for evolutionary branching in a single population. The formation of hybrids of low fitness with intermediate phenotype indeed prevents the trait distribution to split.

In section 3.3, we compare the results we get with the above two-resource model with a second ecological model assuming a continuous distribution of resources [Roughgarden model, 26]. This model assumes a density-dependent logistic growth with carrying capacity $K(u)$ for individuals of trait $u$, a Gaussian function of $u$ with standard deviation $\sigma_{K}$. Competition is also frequency-dependent: individuals with traits $u$ and $u^{\prime}$ compete according to a Gaussian kernel $C\left(u-u^{\prime}\right)$ with standard deviation $\sigma_{C}$. An isolated population evolves to an EBP if $\sigma_{C}<\sigma_{K}$, and to a CSS otherwise [6]. We use a stochastic, sexual version of this model, assuming a polygenic trait $u$ whose transmission occurs as described above for the two-resource model.

\section{$2.3 \quad$ Numerical methods}

Resources dynamics are assumed to be fast compared to the consumer dynamics $\left(\delta_{i} \gg d\right)$. We thus consider that resources are always at their quasi-steady state levels [4]. The birth and death process is simulated as follows. We pick the time until the next event from an exponential distribution with mean the inverse of the total rate at which events occur (sum of individual birth rates $\beta(u)$ and death rates $d$ ). The occurring event is randomly chosen proportionally to the rate of each possible event (birth or death). When an individual is chosen to reproduce, a potential sexual partner is randomly picked. Potential partners are of the opposite sex and their type (resident or immigrant) is chosen according to the premating isolation pattern.

We assume that migration is a rare event. Consequently, we do not allow more than one immigrant population to exist at the same time. Conditional on non-extinction of the total population, the number $X$ of migration attempts before successful residentimmigrant coexistence follows a geometric distribution with mean $1 / p$ and variance $(1-$ $p) / p^{2}$, where $p$ is the probability of stable coexistence after one migration event. For each set of parameter values, we ran 50 replicates. The probability $p$ is estimated by $\hat{p}=1 / \bar{X}$ where $\bar{X}$ denote the mean of $X$ over replicates. The $95 \%$ confidence interval $[\hat{p}-1 /(\bar{X}+e) ; \hat{p}+1 /(\bar{X}-e)]$ where $e=1.96 \sqrt{(1-\hat{p}) /\left(n \hat{p}^{2}\right)}$ are indicated on figures. We consider that resident-immigrant coexistence is stable if both populations are still present after some a priori chosen duration. This duration is chosen long enough to let character displacement be achieved and maintained.

\section{Results}

By running very long simulations without migration event, we checked that we simulate a stable resident population. As expected, the mean trait value reaches the singular point $u^{*}$ (CSS and EBP are convergent stable), and then drifts around the singular point indefinitely, because $u^{*}$ is evolutionarily stable when it is a CSS, and because the formation of hybrids of low fitness prevents the population from evolutionary branching when $u^{*}$ is an EBP.

\subsection{Character displacement under disruptive selection}

As expected, stable coexistence of resident and immigrant populations (i.e. on evolutionary time scales) was always associated with character displacement. The mean trait of 
both populations then diverges on both sides of $u^{*}$ : one is smaller, the other is higher. In other words, the two populations escape from competitive exclusion when they manage to avoid strong competition by specializing on different resources.

When the singular point $u^{*}$ is a CSS, we never obtain successful coexistence (Figure 2). Either immigrants rapidly go extinct or replace residents. A CSS is indeed a fitness maximum: in a resident population with trait $u^{*}$, all mutants have a negative (or zero) fitness, i.e. every other strategy is selected against. Character displacement is thus impossible, resident-immigrant coexistence as well.

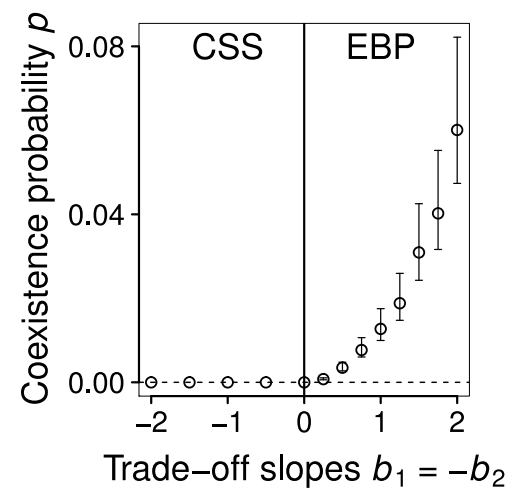

Figure 2: Singular point type effect and strength of disruptive selection effect. Probability $p$ of stable coexistence of resident and immigrant populations after one migration attempt vs absolute values of both slopes $b_{1}$ and $b_{2}$ of the trade-off on resources (Equation 1 ) with $b_{1}=-b_{2}$. When $b_{1} \leq 0$ and $b_{2} \geq 0$, the singular point $u^{*}$ is a CSS. We never observed character displacement in this case. We stopped simulations after 2 millions of generations, which corresponds to a minimum of 10,000 migration attempts. We plotted the corresponding coexistence probability $p$ (necessarily less than $10^{-4}$ ) as zero. When $b_{1}>0$ and $b_{2}<0$, the singular point $u^{*}$ is an EBP. Resident-immigrant coexistence is possible in this case. Increasing the absolute values of the slopes of the trade-off on resources with $b_{1}=-b_{2}$ allows to increase disruptive selection at the singular point $u^{*}$, and hence probability of resident-immigrant coexistence. Parameter values: $V=25$ (equilibrium population size $\approx 1000$ ), $N_{m}=40, \sigma_{m}=0.02, a_{1}=a_{2}-b_{1}, a_{2}=2.1$, $K_{1}=K_{2}=2, k_{1}=k_{2}=1, \delta_{1}=\delta_{2}=1, d=0.1, u^{*}=0.5, \mu=0.01, \sigma_{\mu}=0.02, \pi=1, d_{u}=0$, $\sigma_{b}=0.01$.

On the contrary, when the singular point $u^{*}$ is an EBP, character displacement is possible (Figure 2). This point is a fitness minimum: disruptive selection presses to two specialized strategies on both sides of $u^{*}$. Resident and immigrant populations do not interbreed (or only a little), thus hybrids of intermediate trait are too infrequent to prevent branching of the trait distribution. Consequently the mean resident trait evolves towards a higher or smaller value than $u^{*}$, the mean immigrant trait to the opposite direction (Figure 3). Disruptive selection drives this character displacement, and then maintains specialization: Figure 3 shows that both branches lie on a maximum of the fitness landscape. Note that the process of character displacement lasts only a few hundreds of generations (assuming a mutation probability $\mu=0.01$ and a variance of the distribution of mutants $\sigma_{\mu}=0.02$; character displacement timescale is proportional to $\mu$ and $\left.\left(\sigma_{\mu}\right)^{2}\right)$.

\subsection{Probability of character displacement}

Even if character displacement in sympatry is possible when $u^{*}$ is an EBP, it is far from being certain. Figure 3 shows for example three failed migration attempts. Moreover the variance of the number of migration events before successful resident-mutant coexistence is 

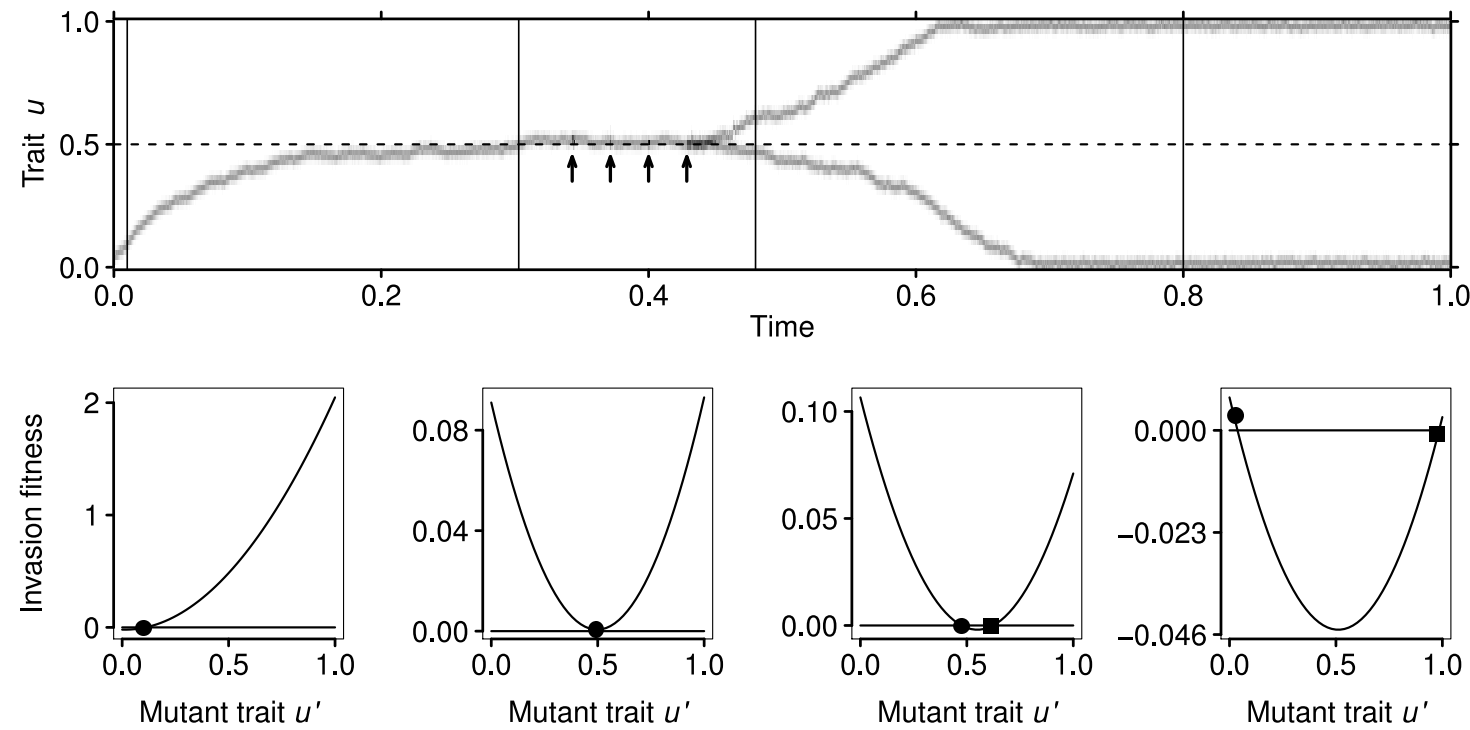

Figure 3: Character displacement under disruptive selection. Typical time series when the singular point $u^{*}$ is an EBP. The dashed line indicates $u^{*}=0.5$. Gray density corresponds to the density of individuals (residents and immigrants summed) of each phenotype. The duration of the simulation is 17,500 generations. Migration events occur at arrows. The fourth migration attempts successfully drives character displacement. Graphs under the time series are the fitness landscape at times indicated by vertical lines. They give the invasion fitness of a rare mutant of trait $u^{\prime}$ in the resident population at these times. Circles (resp. squares) indicate the mean trait and fitness of the resident (resp. immigrant) population. First graph: directional selection to $u^{*}$. Second graph: EBP; the fitness of the population sits at a minimum, allowing for character displacement under disruptive selection to happen. Third graph: character displacement at its beginning; fitness associated to the generalist strategy 0.5 and to the weakly specialized residents and immigrants are close. Fourth graph: character displacement ended; immigrant and resident populations lie on a fitness maximum. Parameter values: $N_{0}=10, u_{0}=0.05$, $V=25$ (equilibrium population size $\approx 1000$ ), $N_{m}=40, d_{u}=0, \sigma_{m}=0.02, a_{1}=0.1, b_{1}=2$, $a_{2}=2.1, b_{2}=-2, K_{1}=K_{2}=2, k_{1}=k_{2}=1, \delta_{1}=\delta_{2}=1, d=0.1, \sigma_{b}=0.01, \mu=0.01$, $\sigma_{\mu}=0.02, \pi=1$.

rather high. Three elements establish the success or failure of migration: (i) the residentimmigrant hybridization pattern, (ii) the efficiency of disruptive selection, and (iii) the strength of drift.

Figure 4 shows that character displacement is likely only for very strong level of premating isolation between resident and immigrant populations. As resident-resident hybrids prevent the resident population from evolutionary branching, resident-immigrant hybrids prevent the two populations from diverging. Hybrids have a generalist strategy and their fitness is close to that of residents and immigrants starting their specialization (see third fitness curve in Figure 3). Consequently hybrids mate, almost as frequently as non-hybrids, which reduces the overall specialization. As a result, as soon as character displacement begins, hybrids break it. Note that hybrid formation does not break character displacement once the latter has been established: hybrids' fitness is very low, hence the hybrid population size is negligible, as well as their impact on the trait distribution.

Character displacement in sympatry is easier when disruptive selection is stronger at the time of a migration event, that is, when the competition between individuals at the generalist strategy $u^{*}$ is more intense and competition between specialist individuals weaker. Figure 2 illustrates this statement. An increase in the absolute values of both slopes $b_{1}$ and $b_{2}$ of the trade-off on resources (Equation 1) means an increase of the ratio 


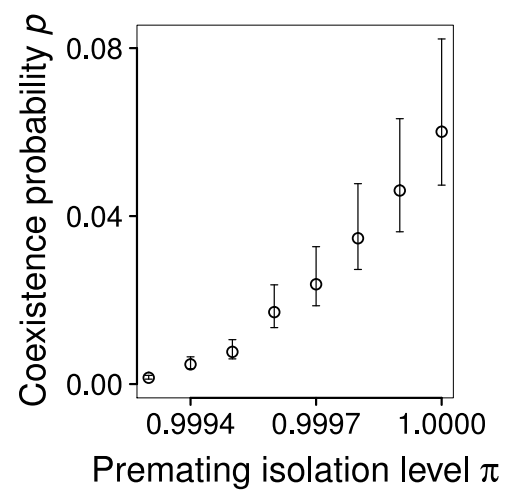

Figure 4: The effect of the level of premating isolation. Probability $p$ of stable coexistence of resident and immigrant populations after one migration attempt vs level of premating isolation $\pi$. When $\pi<1$, offspring type (resident or immigrant) is sexually inherited (see text). Residentimmigrant coexistence probability decreases to very low values for still very strong premating isolation level. Parameter values: $V=25$ (equilibrium population size $\approx 1000$ ), $N_{m}=40$, $d_{u}=0, \sigma_{m}=0.02, a_{1}=0.1, b_{1}=2, a_{2}=2.1, b_{2}=-2, K_{1}=K_{2}=2, k_{1}=k_{2}=1, \delta_{1}=\delta_{2}=1$, $d=0.1, u^{*}=0.5, \sigma_{b}=0.01, \mu=0.01, \sigma_{\mu}=0.02$.

of the efficiency of specialist individuals over generalist individuals. As a result, character displacement becomes more likely. Disruptive selection is also more efficient in a population with a high variance of the trait distribution. Then there are more individuals with extreme phenotypes, whose fitness can be significantly higher than individuals of trait close to $u^{*}$ (see third fitness curve in Figure 3). Consequently they are strongly selected, making character displacement faster and thus easier. Figure 5a illustrates this: a high $\sigma_{b}$ means a strong variance in the trait distribution, so that resident-immigrant coexistence is more likely. Regarding the probability of character displacement, the initial ecological difference $d_{u}$ between residents and immigrants is one of the most important parameters: depending on its value, coexistence can be almost certain or unlikely (Figure 5b). Initiation of character displacement is indeed the most crucial step of character displacement. This can be understood from the fitness curves in Figure 3: the fitness difference between weakly specialized and generalist individuals is much smaller than between fully specialized and generalist individuals. Selection against generalist individuals is thus less efficient at the beginning of character displacement. With a significant initial ecological difference $d_{u}$, disruptive selection only completes and maintains character displacement.

While disruptive selection induces resident-immigrant coexistence, drift impedes it. A migration attempt can fail because immigrants are initially in small number and hence can become extinct just after migration. A migration event is thus more likely to result in character displacement when many individuals migrate at the same time (Figure 6a). If immigrants do not go extinct, stable coexistence can still fail if the immigrants replace the residents. Assuming a weak initial ecological difference between residents and immigrants $\left(d_{u} \approx 0\right)$, resident and immigrant fitnesses are approximately equal. Then, if character displacement takes a long time to happen (due to weak disruptive selection), drift is likely to result in "fixation", i.e., extinction of either all immigrants or all residents. Immigrants can thus replace residents purely due to drift. Figure $6 \mathrm{~b}$ illustrates the effect of drift by showing that character displacement is less likely in a small population (low $V$ ) than in a big one (high $V$ ). 

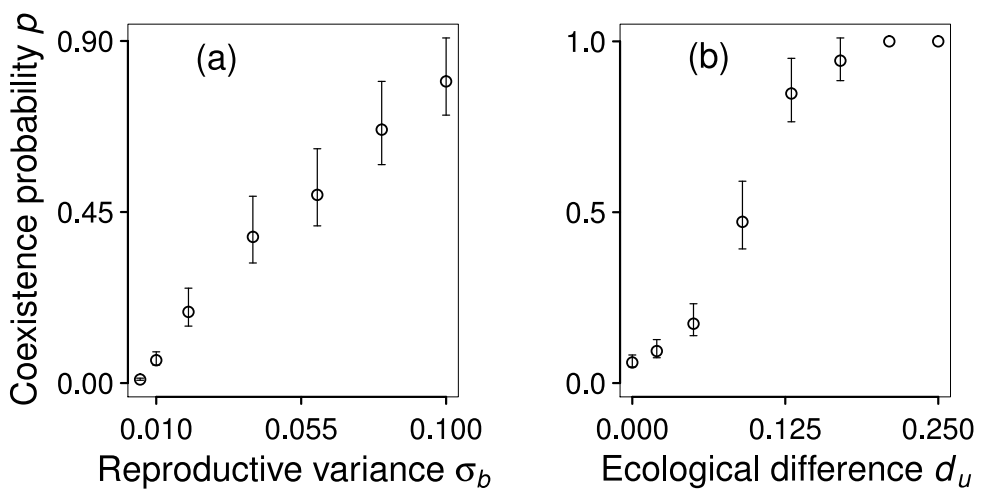

Figure 5: The effect of the trait distribution. (a) When the variance $\sigma_{b}$ in the offspring trait distribution is high, individuals of extreme phenotype are frequently formed. They are strongly selected, which speeds up character displacement making it more likely (and even very likely for high $\sigma_{b}$ ). (b) A moderate initial ecological difference $d_{u}$ between residents and immigrants induces a particularly high coexistence probability. The crucial step of character displacement, namely its initiation, is indeed already performed when secondary contact occurs. For $d_{u} \geq 0.21$ all simulations showed successful coexistence at the first migration attempt. Parameter values: $V=25$ (equilibrium population size $\approx 1000$ ), $N_{m}=40, \sigma_{m}=0.02, a_{1}=0.1, b_{1}=2, a_{2}=2.1$, $b_{2}=-2, K_{1}=K_{2}=2, k_{1}=k_{2}=1, \delta_{1}=\delta_{2}=1, d=0.1, u^{*}=0.5, \mu=0.01, \sigma_{\mu}=0.02, \pi=1$. (a) $d_{u}=0$. (b) $\sigma_{b}=0.01$.
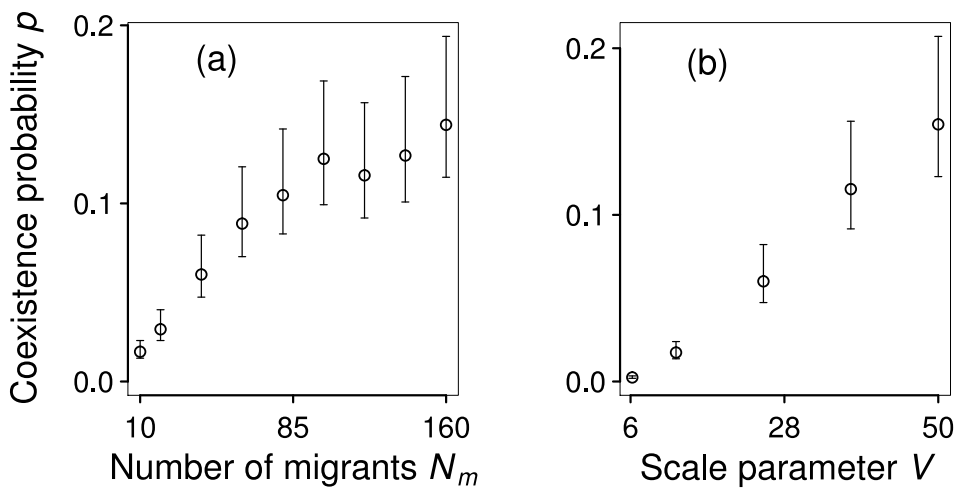

Figure 6: The effect of the strength of drift. (a) Resident-immigrant coexistence is more likely when more immigrants $\left(N_{m}\right.$ migrants) arrive at the same time. The risk of extinction of the immigrant population just after its arrival indeed decreases because it experiences less drift. (b) Coexistence is more likely in a large population (high $V$ ) than in a small one. Parameter values: $d_{u}=0, \sigma_{m}=0.02, a_{1}=0.1, b_{1}=2, a_{2}=2.1, b_{2}=-2, K_{1}=K_{2}=2, k_{1}=k_{2}=1, \delta_{1}=\delta_{2}=1$, $d=0.1, u^{*}=0.5, \sigma_{b}=0.01, \mu=0.01, \sigma_{\mu}=0.02, \pi=1$. (a) $V=25$ (equilibrium population size $\approx 1000$ ). (b) $N_{m}$ varies with $V$ such that the ratio of immigrant over resident is kept at 0.04. The equilibrium population size varies from about 250 to 2000 for $V$ varying from 6 to 50 .

\subsection{Robustness of the results to the ecological model}

To check that our results only depend on the secondary contact scenario and not on the ecological model, we proceed with the same analysis with the continuous-resource model. Results are qualitatively similar: (i) character displacement is possible only if the singular point $u^{*}$ is an EBP (i.e. $\sigma_{C}<\sigma_{K}$ ), (ii) very strong reproductive isolation is needed, and (iii) the probability of character displacement depends on a balance between disruptive selection efficiency and intensity of drift. Quantitatively, however, for analogous sets of parameter values, coexistence probability is usually a bit higher with the continuousresource model than with the two-resource model (not shown). There are indeed only two 
ecological niches in the two-resource model compared to a theoretically infinite number of niches in the continuous-resource model. Character displacement is consequently easier with a continuum of resources. Also because of this difference in niche availability, a third type (and more) can settle in the continuous-resource model whereas only two specialized types can coexist with the two-resource model.

\section{Discussion}

We have shown that sympatric character displacement in two ecologically similar populations is a mechanism that prevents competitive exclusion under two restrictive conditions. First populations should be stuck at a fitness minimum, i.e. at an EBP. Second premating isolation between the two populations should be very strong. We have also shown that character displacement is easier when disruptive selection is strong due to intense competitive interactions; when the variance in the offspring trait distribution is high; when ecological divergence has been initiated in allopatry; and when immigrant and/or resident populations are large. Below we argue that these necessary and promoting conditions are likely to be met in natural populations. Note that using two different ecological models and large ranges of parameter values, we have also shown that our results are robust; they do not depend on the ecological details assumed.

While coexistence of resident and immigrant population on an ecological time scale is a prerequisite for character displacement, their coexistence on an evolutionary time scale is a consequence of character displacement. In particular, when the resident is at a CSS, the coexistence of ecologically differentiated immigrants and residents may be possible, but convergent evolution of residents and immigrants will eventually result in the extinction of one of the populations through competitive exclusion. Only when the resident is at an EBP is coexistence probable on both ecological and evolutionary time scales, the latter mediated by character displacement.

An increasing number of field studies have shown that EBPs are not rare [29]. When an EBP exists, populations are expected to converge to it since an EBP is convergent stable [9]. Populations may find different solutions to escape from this fitness minimum [27]: evolutionary branching, which solved theoretical issues regarding sympatric speciation [6], the evolution of sexual dimorphism [2], the evolution of genetic polymorphism [23, 5], and the evolution of dominance which allows the emergence of specialists [7]. We have characterised here another solution: the migration of a reproductively isolated population, leading to character displacement.

This last scenario requires that the population remains at an EBP until a migration event (i.e. that the other above mechanisms do not act). This can happen for essentially two reasons. First, a population can stay at an EBP without being able to split into two branches because of genetic constraints. Waxman \& Gavrilets [32] stressed that this should be the case for traits under polygenic control. Traits establishing the ecological niche of a population are usually complex, so that it is not senseless to think that many genes are involved. In the quite simple case of Darwin's finches, beak size and shape set the ecological niche through diet. Polygenic control is known for these traits [15]. Second, a population can be locked on an EBP because of small population size $[4,5]$. Demographic stochasticity is significant in small populations, which delays evolutionary branching. It may be the case for Darwin's finches. Grant et al. [20] evaluate that the finch population size of two species ( $G$. fortis and G. scandens) on the island Daphne Major varies from 300 to 3,000 individuals (our simulations assume 1,000 individuals). This is a small enough population size to observe delayed evolutionary branching according to Claessen et al.'s studies. 
In agreement with Grant \& Grant [11, 15], we have assumed sexual imprinting of mating preferences for resident-immigrant hybrids. However the result that very strong premating isolation is required for character displacement does not rest on this hypothesis: as soon as individuals of intermediate phenotype exist (hybrids), character displacement is prevented. Field studies already pointed out the necessity of strong premating isolation. In the case of Darwin's finches, some of them demonstrate the existence of premating isolation [e.g. 12] whereas many others attest hybrid formation [e.g. 10, 14, 15, 16, 19]. It seems thus reasonable to think that assortative mating is not absolute at the time of secondary contact, but that it may evolve through reinforcement. Reinforcement should be fast enough so that the immigrant population does not go extinct or does not replace the resident population. Reinforcement can result from selection against hybrids that carry genetic incompatibilities reducing their fitness (intrinsic reinforcement). Grant \& Grant [14] however demonstrated no genetic loss of fitness in Darwin's finches hybrids. Here we postulate reinforcement due to increased competition generated by hybrid formation, i.e. extrinsic ecological reinforcement [22, 29].

Regarding the initial ecological divergence at the time of secondary contact, our simulations assume the most constraining hypothesis: no ecological divergence. We have however shown that a small ecological difference is enough to significantly increase the probability of character displacement. In this case, populations do not occupy exactly the same niche: they have almost already avoided competitive exclusion. Allopatric populations spreading into sympatry are often known already to have initiated an ecological divergence in allopatry due to different local environmental and/or ecological conditions[29, 28, 30].

We have shown that character displacement may occur in only a few hundreds of generations. As a result, even if character displacement is unlikely, considering a reasonable timescale, we can expect many secondary contacts before one of them successfully leads to character displacement. For example, Darwin's finches radiation has lasted for 2.8 million years [13]. Numerous migration events could have occurred before at least one successful character displacement. Moreover we may significantly overestimate the time needed for character displacement: Grant \& Grant [17] directly observed it on Daphne Major island in a period of time of only 22 years.

We have focused on Darwin's finches because their radiation scenario is well-documented, allowing us some comparisons with our results. Note however that our model may allow to better understand speciation in other species as well. As already mentioned, the same scenario is thought to take place in many nonadaptive radiations [28]. Moreover, ring species, which consist of two reproductively isolated populations connected by a chain of interbreeding populations, are considered as the continental analogues of the Galápagos finches $[15,21]$. The two reproductively isolated terminal populations that come into secondary contact may be ecologically similar. Competitive interactions may result in competitive exclusion. Ecological interactions could then be a source of disruptive selection.

Finally, we should mention that our model implicitly assumes constant environmental conditions. This should not be the case over a long period of time. For example, during Darwin's finches radiation, global cooling and warming periods alternated, some islands in the Galápagos archipelago have been submerged, others emerged [3]. It would thus be valuable to incorporate in our model a dynamic landscape framework [1], that is, the opportunity for several populations to merge and split repeatedly. Such dynamic landscapes may lead to repetitive character displacement in a dynamical equilibrium. Populations may then avoid competitive exclusion under much broader environment conditions. 


\section{Acknowledgments}

R. A. is supported by a PhD fellowship from the French Ministère de la Recherche et de la Technologie. Traveling was funded by the project Modèles Aléatoire de l'Evolution du Vivant (MAEV) of the French Agence Nationale pour la Recherche (ANR-06-BLAN3_146282). We thank Marianne Mugabo for helpful comments on previous versions of the manuscript.

\section{References}

[1] R. Aguilée, D. Claessen, and A. Lambert, Allele fixation in a dynamic metapopulation: founder effects vs refuge effects., Theor Popul Biol 76 (2009), pp. 105-117.

[2] D.I. Bolnick and M. Doebeli, Sexual dimorphism and adaptive speciation: two sides of the same ecological coin., Evolution 57 (2003), pp. 2433-2449.

[3] D. Christie, R. Duncan, A. McBirney, M. Richards, W. White, K. Harpp, and C. Fox, Drowned islands downstream from the Galapagos hotspot imply extended speciation times, Nature 355 (1992), pp. 246-248.

[4] D. Claessen, J. Andersson, L. Persson, and A.M. Roosde , Delayed evolutionary branching in small populations, Evol Ecol Res 9 (2007), pp. 51-69.

[5] D. Claessen, J. Andersson, L. Persson, and A.M. Roosde, The effect of population size and recombination on delayed evolution of polymorphism and speciation in sexual populations, Am Nat 172 (2008), pp. E18-E34.

[6] U. Dieckmann and M. Doebeli, On the origin of species by sympatric speciation, Nature 400 (1999), pp. 354-357.

[7] M. Durinx and T.J.M. Doorenvan, Assortative mate choice and dominance modification: alternative ways of removing heterozygote disadvantage., Evolution 63 (2009), pp. 334-352.

[8] G.F. Gause, The Williams \& Wilkins company, Baltimore 1934.

[9] S. Geritz, E. Kisdi, G. Meszéna, and J. Metz, Evolutionarily singular strategies and the adaptive growth and branching of the evolutionary tree, Evol Ecol 12 (1998), pp. $35-57$.

[10] B.R. Grant and P.R. Grant, Darwin's finches: population variation and sympatric speciation, Proc Natl Acad Sci USA 76 (1979), pp. 2359-2363.

[11] B.R. Grant and P.R. Grant, Cultural inheritance of song and its role in the evolution of Darwin's finches, Evolution 50 (1996), pp. 2471-2487.

[12] B.R. Grant and P.R. Grant, Simulating secondary contact in allopatric speciation: an empirical test of premating isolation, Biol J Linn Soc 76 (2002), pp. 545-556.

[13] P.R. Grant and B.R. Grant, Phenotypic and genetic effects of hybridization in Darwin's finches, Evolution 48 (1994), pp. 297-316.

[14] P.R. Grant and B.R. Grant, Speciation and hybridization in island bird, Philos Trans R Soc Lond B Biol Sci 351 (1996), pp. 765-772. 
[15] P.R. Grant and B.R. Grant, Genetics and the origin of bird species, Proc Natl Acad Sci USA 94 (1997), pp. 7768-7775.

[16] P.R. Grant and B.R. Grant, Hybridization, sexual imprinting, and mate choice, Am Nat 149 (1997), pp. 1-28.

[17] P.R. Grant and B.R. Grant, Evolution of character displacement in Darwin's finches, Science 313 (2006), pp. 224-226.

[18] P.R. Grant, B.R. Grant, and K. Petren, The allopatric phase of speciation: the sharpbeaked ground finch (Geospiza difficilis) on the Galápagos islands, Biol J Linn Soc 69 (2000), pp. 287-317.

[19] P.R. Grant, B.R. Grant, and K. Petren, Hybridization in the recent past, Am Nat 166 (2005), pp. 56-67.

[20] P.R. Grant, B.R. Grant, L. Keller, and K. Petren, Effects of El Nino events on Darwin's finch productivity, Ecology 81 (2000), pp. 2442-2457.

[21] D. Irwin, J. Irwin, and T. Price, Ring species as bridges between microevolution and speciation, Genetica 112 (2001), pp. 223-243.

[22] M. Kirkpatrick, Reinforcement during ecological speciation., Proc R Soc Lond B Biol Sci 268 (2001), pp. 1259-1263.

[23] E. Kisdi and S.A.H. Geritz, Adaptive dynamics in allele space: evolution of genetic polymorphism by small mutations in a heterogeneous environment, Evolution 53 (1999), pp. 993-1008.

[24] J. Metz, S. Geritz, G. Meszéna, F. Jacobs, and J. van Heerwaarden, Adaptive dynamics: a geometrical study of the consequences of nearly faithful reproduction, in Stochastic and spatial structures of dynamical systems, S.J. van Strien and S.M.V. Lunel, eds., Amsterdam: North-Holland, 1996, pp. 183-231.

[25] L.M. Ratcliffe and P.R. Grant, Species recognition in Darwin's finches (Geospiza, Gould). I. Discrimination by morphological cues, Anim Behav 31 (1983), pp. 11391153.

[26] J. Roughgarden, Evolution of niche width, Am Nat 106 (1972), pp. 683-718.

[27] C. Rueffler, T.J.M. Doorenvan , O. Leimar, and P.A. Abrams, Disruptive selection and then what?, Trends Ecol Evol 21 (2006), pp. 238-245.

[28] R.J. Rundell and T.D. Price, Adaptive radiation, nonadaptive radiation, ecological speciation and nonecological speciation, Trends Ecol Evol 24 (2009), pp. 394-399.

[29] H.D. Rundle and P. Nosil, Ecological speciation, Ecol Lett 8 (2005), pp. 336-352.

[30] D. Schluter, Evidence for ecological speciation and its alternative, Science 323 (2009), pp. $737-741$.

[31] D. Schluter, T.D. Price, and P.R. Grant, Ecological character displacement in Darwin's finches., Science 227 (1985), pp. 1056-1059.

[32] D. Waxman and S. Gavrilets, 20 questions on adaptive dynamics, J Evol Biol 18 (2005), pp. 1139-1154. 